\title{
Methylphenidate Misuse in Adults: Survey of 414 Primary Care Physicians in Germany and Comparison with the Literature*
}

\author{
Ria Thier, Ursula Gresser \\ Ludwig Maximilians University of Munich, Medical Faculty, Internal Medicine, Munich, Germany \\ Email:ursulagresser@email.de
}

How to cite this paper: Thier, R. and Gresser, U. (2017) Methylphenidate Misuse in Adults: Survey of 414 Primary Care Physicians in Germany and Comparison with the Literature. Journal of Behavioral and Brain Science, 7, 180-209. https://doi.org/10.4236/jbbs.2017.74015

Received: February 20, 2017

Accepted: April 15, 2017

Published: April 18, 2017

Copyright ( 92017 by authors and ScientificResearch Publishing Inc. This work is licensed under the CreativeCommons Attribution International

License (CC BY 4.0).

http://creativecommons.org/licenses/by/4.0/

(c) (i) Open Access

\begin{abstract}
Objectives: This paper deals with the methylphenidate (MPH) misuse by adults in Germany. Results of a survey among primary care physicians/internists have been supplemented by a comparison with the literature. Methods: In the period from October 5-20, 2015, a survey was sent to 414 primary care physicians/internists in four German cities $(\mathrm{n}=10$ were undeliverable). The response rate was $58 \%(\mathrm{n}=235)$. 34 original works on MPH abuse worldwide were found in the literature and are used in the analysis of the present data situation. The literature published before November 9, 2015 is considered in this paper. Results: $14 \%$ of the doctors who took part in the survey said that they had been asked for MPH prescriptions without any medical indication. The most frequent reason given for the request (42\%) was adult attention deficit/hyperactivity disorder (ADHD) not verified by documents. According to the comparison with the literature, university students had a lifetime prevalence of MPH misuse ranging from $0.8 \%$ to $16.6 \%$ and school children had a misuse rate of $4.0 \%$. In the civilian US population, the misuse rate was $4.2 \%$. Among patients in possession of a current MPH prescription due to a diagnosis, the lifetime prevalence was $29 \%$ and among adolescents with suspected alcohol and/or drug problems 20\%. Conclusions: MPH misuse is a major problem which has not been studied sufficiently. MPH misuse not only plays a role in the field of psychiatry, but also in other disciplines. Misuse particularly following a therapeutic prescription should be taken into account.
\end{abstract}

\section{Keywords}

Methylphenidate, Misuse, Students, Prescription Drugs

${ }^{\star}$ The results published are part of the dissertation by RiaThier on methylphenidate misuse in adults (Ludwig MaximiliansUniverstity of Munich, Germany; in preparation) 


\section{Introduction}

In Germany, methylphenidate (MPH) came on the market in 1954 [1]. After it was available without prescription for years, it became subject to the law governing the use and traffic of drugs in 1971 [2]. The substance acts-similarly to amphetamine and methamphetamine-as an inhibitor on presynaptic neurotransmitter transporters, especially on transporters of dopamine and noradrenaline [3] [4].

The principal application is the treatment of attention deficit/hyperactivity disorder (ADHD) among children and since 2011 a drug containing methylphenidate has been available for the treatment of adults [5] [6]. As a conceivable result, the number of insured employed people with at least one MPH prescription increased in Germany by $80 \%$ in 2013 compared to 2011 [7]. The largest increase in terms of percentage (148\%) was found among patients in their early 30's [7].

A look at the side effects reveals that the substance should not be underestimated. According to a triple-blind, placebo-controlled study, a low-dose MPH intake $(0.3 \mathrm{mg} / \mathrm{kg})$ may already cause unspecific side effects such as lack of appetite, insomnia, stomach pains and headaches [8]. Moreover, intake can produce tachycardia as well as a diastolic and systolic hypertension [9]. An overdose is noticeable by numerous-predominantly cardiovascular and neurological-sympathomimetic effects [10]. Typical cardiovascular indicators of an overdose are tachyarrhythmias [10]. Neurological complaints are sometimes harmless irritability or euphoria; however, they can also result in psychosis and epileptic seizure [10]. According to a recent review study from our group of authors, a MPH therapy may even entail-potentially persistent-changes in behavior [11].

In recent years, there have been multiple indications of adult drug abuse of methylphenidate, and it is usually mentioned in the context of desired performance enhancement [12] [13] [14]. In this regard, methylphenidate is-with regard to prescription drugs in general-the most known stimulant within the group of German students [13]. Considering that $90 \%$ of German students feel stressed occasionally or frequently, the desire for performance enhancement does not appear far-fetched [15]. The authors of the present study used the following definition for methylphenidate misuse/abuse: recreational use and/or use without a prescription [16]. Because the current literature does not allow any clear separation between "misuse" and "abuse", these two terms are considered as synonymous in this paper. However, in the following, both terms are used to ensure that recent studies are cited properly.

The aim of this paper was to investigate the role of methylphenidate misuse among adults in Germany. It intended to detect whether this circumstance plays a role in general and-if so-in which order of approximate magnitude. As far as the authors know, this is the first survey of German doctors/internists on the subject of methylphenidate abuse among adults and should serve as a pilot study for potential following representative data collection. Previous reviews focused mainly on the US population and on stimulants in general; in contrast, the 
present paper focuses on misuse world-wide and on methylphenidate, in particular [17].

\section{Methods}

\subsection{Participants and Procedure}

From the October 5-24, 2015, questionnaires on problem were sent to primary care physicians/internists who treat non-privately insured patients ${ }^{1}$ and practice in four German cities. Even though we did not aim to obtain representative data, we have selected four cities with different population structures, geographic locations in the country and sizes-instead of one-in order to obtain certain extent reliable, transferable data. Selected were Munich (International city, population $\sim 1.5$ million), Frankfurt am Main (Financial metropolis, population $\sim 732,000$, formerly Western Germany), Augsburg (Rural area, population 287,000) and Halle an der Saale (Formerly Eastern Germany, 237,000 citizens). If differences within the cities were noteworthy, they naturally were given attention. The contact addresses are public data from the Association of Statutory Health Insurance Physicians (www.kvb.de, www.kvhessen.de, www.kvsa.de), and the authors received an alphabetical address list for each city with the entire group of doctors who treat non-privately insured patients in the respective city (Munich, Frankfurt am Main and Augsburg on August 31, 2015, Halle an der Saale on September 1, 2015). Every doctor on the list for Frankfurt am Main ( $\mathrm{n}=132)$, Augsburg $(\mathrm{n}=55)$ and Halle an der Saale $(\mathrm{n}=101)$ was contacted. To receive rough similar and proportionate list sizes per city, instead of a random sample, exactly only every fourth doctor from the strictly alphabetical list for Munich (no. 1, 5, 8, $13, \ldots$ : thus $n=216$ ) was contacted. In total, 414 primary care physicians/internists were asked to participate in the survey. Apart from the requirement for inclusion on those lists, there were no inclusion or exclusion criteria defined.

\subsection{Questionnaire}

In addition to the questionnaire, all envelopes contained a personal cover letter and a handwritten self-addressed stamped envelope. The cover letter did not include a specific consent form. Instead respondents were given the option of stating their names or remaining anonymous, thus a non-participation was evaluated as non-consent in general, the decision for an anonymous participation as consent for an anonymous participation and the decision for a participation by stating their name as consent for a participation by name. It took at least five minutes to answer the following nine questions (Question 1 to 3: corresponding wording, cf. Supplemental data for precise wording):

1. Are you a primary care physician, an internist or something different?

2. Are you practicing in Munich/Frankfurt/Augsburg/Halle an der Saale?

3. Are you working in the practice since 1 to 5 years (since 2011), 5 to 10 years (before 2010), 10 to 15 years (before 2005), 16 to 20 years (before 2000), 21 years and longer (before 1995)?

4. Do you have patients in your care with prescribed methylpenidate for medical ${ }^{1}$ Participant in the treatment of non-privately insured patients $=$ panel doctor $=$ contract doctor 
reasons?

5. Have you ever had a case in which you were asked to prescribe methylpenidate without medical indication?

6. If so: Who asked and who was it for?

7. What was the reason given-from your point of view-for requesting a prescription without indication?

8. What did you do when asked?

9. Do you want to add something related to the topic?

As mentioned earlier, the survey is intended to provide an overview and not representative data, thus we accepted the use of an invalidated questionnaire. No approval of the local ethics committee was needed, thus an anonymous data collection without patient data was used.

$97 \%(n=404)$ of the envelopes were delivered by mail and 235 persons took part. This represents a response rate of $58 \%$ (cf. Table 1 ). Nine questionnaires could not be considered in the analysis due to a blank sheet $(n=6)$ or inconsistent information $(n=$ 3), meaning that 226 respondents were evaluated. Among these respondents, the majority (59\%) stated their names and provided the authors with additional contact data. 99\% of the surveyed doctors said they were internists. Questioned in regard to the number of years the doctors have been practicing at the time of the study, there was an almost homogeneous distribution with one year to 21 or more years. Additional questions related to personal characteristics were rejected, fearing that this information increase at the expense of participants and honest answers. Participants may fear criminal investigations.

Assuming that a primary care doctor sees about 991 patients per quarter (eg. based on data for the fourth quarter of 2015: Bavaria: 883; Hesse: 909; Saxony-Anhalt: 1,090) and the analysis below included 226 survey participants, the survey considered 217,186 treated cases in just one quarter [18] [19] [20].

\subsection{Literature Search}

For the comparison with the latest studies on methylphenidate misuse the key words for the literature search were as follows: "methylphenidate misuse", "methylphenidate abuse", "methylphenidate neuroenhancement", "methylphenidate students", "ritalin misuse", "ritalin abuse", "ritalin neuroenhancement", "ritalin students". The following databases were used: PubMed (www.ncbi.nlm.gov/pub med), Google (www.google.de) and Google Scholar (www.scholar.google.de).

Table 1. Responses to the questionnaire. $96.2 \%(n=226)$ of the respondents stated the city.

\begin{tabular}{cccc}
\hline Town & Dispatched & Delivered & Response rate \\
\hline Munich & $30.4 \%(\mathrm{n}=126)$ & $96.8 \%(\mathrm{n}=122)$ & $60.7 \%(\mathrm{n}=74)$ \\
Frankfurt & $31.9 \%(\mathrm{n}=132)$ & $97.0 \%(\mathrm{n}=128)$ & $50.0 \%(\mathrm{n}=64)$ \\
Augsburg & $13.3 \%(\mathrm{n}=55)$ & $98.2 \%(\mathrm{n}=54)$ & $61.1 \%(\mathrm{n}=33)$ \\
Halle & $24.4 \%(\mathrm{n}=101)$ & $99.0 \%(\mathrm{n}=100)$ & $53.0 \%(\mathrm{n}=53)$ \\
Total & $100 \%(\mathrm{n}=414)$ & $97.6 \%(\mathrm{n}=404)$ & $58.2 \%(\mathrm{n}=235)$ \\
\hline
\end{tabular}


Only original works on the subject of methylphenidate misuse were considered for this study. Studies dealing with stimulants in general were only considered on condition that cases of methylphenidate misuse were also mentioned. In total, 34 original works were considered for this study. The search cutoff date was November 9, 2015. Later publications are not included in this paper.

\section{Results}

\subsection{Part 1: Survey in Germany}

\subsubsection{Patients with Medical Indication for Use of Methylphenidate}

With the exception of one individual, all respondents provided information on their care of patients having a medical indication for methylphenidate. Almost $25 \%$ of the respondents gave a positive answer. $75 \%$ of them said that their patients were adults and $53 \%$ had children under their care. When comparing the four cities the greatest difference was between Munich and Halle (cf. Table 2).

\subsubsection{Inquiries with Regard to Non-Indicated Methylphenidate Prescriptions}

$14 \%$ of the respondents had already been confronted with a situation in which they were asked to prescribe methylphenidate without a medical indication. The highest prevalence was in Munich (31\%) and the lowest in Halle (4\%). One doctor from Munich (no. 1) added a handwritten note ("or I wasn't aware of it") in reference to additional unperceived cases, and one doctor from Frankfurt (no. 181) was certain concerning this regard ("definitely one female addict") (cf. Table 3). When evaluating the questionnaires, it was assumed the doctors who checked "No, I have never had that, but I have heard of such cases from colleagues" also marked "No, I have never encountered it" as well. 44\% ( $n=14)$ of

Table 2. Question: “Have you prescribed methylphenidate for medical reasons?". 99.6\% $(\mathrm{n}=225)$ responded. Partly multiple answers.

\begin{tabular}{|c|c|c|c|c|}
\hline \multirow{2}{*}{ City } & \multirow{2}{*}{ No } & \multicolumn{3}{|c|}{ Yes } \\
\hline & & Children & Adults & Both \\
\hline $\begin{array}{l}\text { Munich } \\
(\mathrm{n}=74)\end{array}$ & $60.8 \%(n=45)$ & $48.3 \%(\mathrm{n}=14)$ & $\begin{array}{l}39.2 \%(n=29) \\
65.5 \%(n=19)\end{array}$ & $13.8 \%(\mathrm{n}=4)$ \\
\hline $\begin{array}{c}\text { Frankfurt } \\
(\mathrm{n}=64)\end{array}$ & $85.9 \%(n=55)$ & $55.6 \%(\mathrm{n}=5)$ & $\begin{array}{l}14.1 \%(\mathrm{n}=9) \\
88.9 \%(\mathrm{n}=8)\end{array}$ & $44.4 \%(n=4)$ \\
\hline $\begin{array}{l}\text { Augsburg } \\
(\mathrm{n}=33)\end{array}$ & $81.8 \%(\mathrm{n}=27)$ & $66.7 \%(\mathrm{n}=4)$ & $\begin{array}{l}18.2 \%(n=6) \\
100 \%(n=6)\end{array}$ & $66.7 \%(\mathrm{n}=4)$ \\
\hline $\begin{array}{c}\text { Halle } \\
(\mathrm{n}=52)\end{array}$ & $88.5 \%(n=46)$ & $66.7 \%(\mathrm{n}=4)$ & $\begin{array}{l}11.5 \%(n=6) \\
66.7 \%(n=4)\end{array}$ & $33.3 \%(n=2)$ \\
\hline $\begin{array}{l}\text { Not stated } \\
\quad(\mathrm{n}=2)\end{array}$ & $50 \%(\mathrm{n}=1)$ & $0 \%(n=0)$ & $\begin{array}{l}50 \%(\mathrm{n}=1) \\
100 \%(\mathrm{n}=1)\end{array}$ & $0 \%(n=0)$ \\
\hline $\begin{array}{c}\text { Total } \\
(\mathrm{n}=225)\end{array}$ & $77.3 \%(\mathrm{n}=174)$ & $52.9 \%(\mathrm{n}=27)$ & $\begin{array}{l}22.7 \%(\mathrm{n}=51) \\
74.5 \%(\mathrm{n}=38)\end{array}$ & $27.5 \%(\mathrm{n}=14)$ \\
\hline
\end{tabular}


the doctors who had already encountered a request to prescribe methylphenidate without medical indication answered the previous question by stating that they also treat patients with indicated methylphenidate.

\subsubsection{Frequency of Inquiries}

$63 \%$ of the doctors who stated they had been asked to prescribe methylphenidate without medical indication had encountered the situation three times at maximum. A doctor from Frankfurt was asked regularly about once a month (cf. Table 4).

\subsubsection{Who Asked and Who Was It for?}

The majority of the inquiries (84\%) was adults requesting a prescription for themselves. $29 \%$ of the inquiries were made by adults for their child. Four doctors-three from Munich-were confronted with both situations. In Munich, the number of inquiries "for themselves" $(\mathrm{n}=20)$ was higher than the average (cf. Table 5).

About $50 \%$ of the adults asking for a prescription for themselves were known patients and about $50 \%$ were unknown new patients. No major differences were found between the various cities (cf. Table 6). Three additional notes were made by hand:

Table 3. Question: "Have you ever had a case in which you were asked to prescribe methylphenidate without medical indication?" $100 \%(n=226)$ responded.

\begin{tabular}{cccc}
\hline City & No, I never have & $\begin{array}{c}\text { No, I never have, but I } \\
\text { have colleagues who have }\end{array}$ & Yes, that has happened \\
\hline Munich $(\mathrm{n}=74)$ & $68.9 \%(\mathrm{n}=51)$ & $5.4 \%(\mathrm{n}=4)$ & $31.1 \%(\mathrm{n}=23)$ \\
Frankfurt $(\mathrm{n}=64)$ & $92.2 \%(\mathrm{n}=59)$ & $0 \%(\mathrm{n}=0)$ & $7.8 \%(\mathrm{n}=5)$ \\
Augsburg $(\mathrm{n}=33)$ & $93.9 \%(\mathrm{n}=31)$ & $3.0 \%(\mathrm{n}=1)$ & $6.1 \%(\mathrm{n}=2)$ \\
Halle $(\mathrm{n}=53)$ & $96.2 \%(\mathrm{n}=51)$ & $0 \%(\mathrm{n}=0)$ & $3.8 \%(\mathrm{n}=2)$ \\
Not stated $(\mathrm{n}=2)$ & $100 \%(\mathrm{n}=2)$ & $0 \%(\mathrm{n}=0)$ & $0 \%(\mathrm{n}=0)$ \\
Total $(\mathrm{n}=226)$ & $85.8 \%(\mathrm{n}=194)$ & $2.2 \%(\mathrm{n}=5)$ & $14.2 \%(\mathrm{n}=32)$ \\
\hline
\end{tabular}

Table 4. Frequency, when "Have you ever had a case in which you were asked to prescribe methylphenidate without medical indication?" was answered with "Yes, that has happened". 100\% $(\mathrm{n}=32)$ responded.

\begin{tabular}{|c|c|c|c|c|}
\hline \multirow[b]{2}{*}{ City } & \multicolumn{3}{|c|}{ Frequency } & \multirow{2}{*}{$\begin{array}{r}\text { First time.. } \\
\text { years ago }\end{array}$} \\
\hline & Only once & $\begin{array}{c}\text { In total, three } \\
\text { times at maximum }\end{array}$ & $\begin{array}{c}\text { About once a } \\
\text { month }\end{array}$ & \\
\hline $\begin{array}{l}\text { Munich } \\
(\mathrm{n}=23)\end{array}$ & $30.4 \%(n=7)$ & $69.6 \%(n=16)$ & $0 \%(\mathrm{n}=0)$ & $\begin{array}{c}13 \%(\mathrm{n}=3) \\
{[3 / 7 / 10]}\end{array}$ \\
\hline $\begin{array}{l}\text { Frankfurt } \\
\quad(\mathrm{n}=5)\end{array}$ & $40.0 \%(n=2)$ & $40.0 \%(n=2)$ & $20.0 \%(\mathrm{n}=1)$ & $0 \%(\mathrm{n}=0)$ \\
\hline $\begin{array}{l}\text { Augsburg } \\
(\mathrm{n}=2)\end{array}$ & $100 \%(n=2)$ & $0 \%(\mathrm{n}=0)$ & $0 \%(\mathrm{n}=0)$ & $0 \%(\mathrm{n}=0)$ \\
\hline $\begin{array}{l}\text { Halle } \\
(\mathrm{n}=2)\end{array}$ & $0 \%(\mathrm{n}=0)$ & $100 \%(n=2)$ & $0 \%(n=0)$ & $0 \%(\mathrm{n}=0)$ \\
\hline $\begin{array}{c}\text { Total } \\
(\mathrm{n}=32)\end{array}$ & $34.4 \%(\mathrm{n}=11)$ & $62.5 \%(n=20)$ & $3.1 \%(n=1)$ & $\begin{array}{c}9.4 \%(\mathrm{n}=3) \\
{[3 / 7 / 10]}\end{array}$ \\
\hline
\end{tabular}


Table 5. Follow-up question: "Who asked and who was it for?", if " Have you ever had a case in which you were asked to prescribe methylphenidate without medical indication "was answered with "Yes, that has happened". 96.9\% $(n=31)$ responded. Partly multiple answers.

\begin{tabular}{cccc}
\hline City & An adult asked own use An adult asked in favor of a child & Both \\
\hline Munich $(\mathrm{n}=23)$ & $87.0 \%(\mathrm{n}=20)$ & $26.1 \%(\mathrm{n}=6)$ & $13.0 \%(\mathrm{n}=3)$ \\
Frankfurt $(\mathrm{n}=4)$ & $100 \%(\mathrm{n}=4)$ & $25.0 \%(\mathrm{n}=1)$ & $25.0 \%(\mathrm{n}=1)$ \\
Augsburg $(\mathrm{n}=2)$ & $0 \%(\mathrm{n}=0)$ & $100 \%(\mathrm{n}=2)$ & $0 \%(\mathrm{n}=0)$ \\
Halle $(\mathrm{n}=2)$ & $100 \%(\mathrm{n}=2)$ & $0 \%(\mathrm{n}=0)$ & $0 \%(\mathrm{n}=0)$ \\
Total $(\mathrm{n}=31)$ & $83.9 \%(\mathrm{n}=26)$ & $29.0 \%(\mathrm{n}=9)$ & $12.9 \%(\mathrm{n}=4)$ \\
\hline
\end{tabular}

Table 6. Information relating to the patient status, if an adult asked a prescription for own use. $92.3 \%(n=24)$ responded. Partly multiple answers. The note "Filling in for a colleague" was added in the category "An unknown patient" and the notes "acquaintance!" and "female colleague" in the category "A known patient".

\begin{tabular}{cccc}
\hline City & A known patient & An unknown patient & Both \\
\hline Munich $(\mathrm{n}=19)$ & $47.4 \%(\mathrm{n}=9)$ & $57.9 \%(\mathrm{n}=11)$ & $5.3 \%(\mathrm{n}=1)$ \\
Frankfurt $(\mathrm{n}=3)$ & $66.7 \%(\mathrm{n}=2)$ & $33.3 \%(\mathrm{n}=1)$ & $0 \%(\mathrm{n}=0)$ \\
Augsburg $(\mathrm{n}=0)$ & & & \\
Halle $(\mathrm{n}=2)$ & $100 \%(\mathrm{n}=2)$ & $0 \%(\mathrm{n}=0)$ & $0 \%(\mathrm{n}=0)$ \\
Total $(\mathrm{n}=24)$ & $54.2 \%(\mathrm{n}=13)$ & $50.0 \%(\mathrm{n}=12)$ & $4.2 \%(\mathrm{n}=1)$ \\
\hline
\end{tabular}

A "Filling in for a colleague" (no. 35)

A "Acquaintance!" (no. 73)

A "Female colleague" (no. 113)

$83 \%$ of the adults who requested a methylphenidate prescription for themselves were between 20 and 40 years of age.

Two third of the adults who wanted a prescription for their child were known patients and one third unknown patients. Additionally, one note was added by hand: "a colleague" (no. 52).

\subsubsection{Reasons for Wanting a Prescription}

The most frequent reasons given for a prescription request without indication were "ADHD in adults-but not proved by documents" (42\%) and "The drug is needed for learning and tests" (38\%). In Munich, eleven out of 22 patients requested a prescription due to adult ADHD, not proved by documents (cf. Table 7). Additionally, two notes were added by hand:

$\triangle$ "X) So that he doesn't always fall asleep during the opera" (no. 96)

A "Tiredness during the day" (no. 223)

\subsubsection{How Did Doctors React to This Situation}

Most doctors (65\%) said that, in this situation, they did not prescribe methylphenidate because they would not give prescriptions without indication. About $10 \%$ examined the person. Altogether $29 \%$ issued a prescription to patients (cf. Table 8). Two notes were added by hand regarding "other reasons": 
Table 7. Question: "What was the reason given-from your point of view-for requesting a prescription without indication?”. 90.6\% (n = 29) responded. Partly multiple answers. The note " $\mathrm{x}$ ) so that he doesn't always fall asleep during the opera" is not listed and the note "tiredness during the day" in the category "The medicine is needed to improve the ability to work".

\begin{tabular}{cccccc}
\hline City & $\begin{array}{c}\text { ADHD in } \\
\text { adults-but not } \\
\text { proved by } \\
\text { documents }\end{array}$ & $\begin{array}{c}\text { The primary } \\
\text { care physician } \\
\text { cannot be } \\
\text { reached }\end{array}$ & $\begin{array}{c}\text { The medicine } \\
\text { is needed for } \\
\text { learning and } \\
\text { tests }\end{array}$ & $\begin{array}{c}\text { The medicine is } \\
\text { needed to improve } \\
\text { the ability to work }\end{array}$ & No reason \\
\hline $\begin{array}{c}\text { Munich } \\
(\mathrm{n}=22)\end{array}$ & $50.0 \%$ & $31.8 \%$ & $27.3 \%$ & $18.2 \%$ & $9.1 \%$ \\
$(\mathrm{n}=11)$ & $(\mathrm{n}=7)$ & $(\mathrm{n}=6)$ & $(\mathrm{n}=4)$ & $(\mathrm{n}=2)$ \\
Frankfurt & $0 \%$ & $33.3 \%$ & $66.7 \%$ & $33.3 \%$ & $33.3 \%$ \\
$(\mathrm{n}=3)$ & $(\mathrm{n}=0)$ & $(\mathrm{n}=1)$ & $(\mathrm{n}=2)$ & $(\mathrm{n}=1)$ & $(\mathrm{n}=1)$ \\
Augsburg & $0 \%$ & $0 \%$ & $100 \%$ & $0 \%$ & $0 \%$ \\
$(\mathrm{n}=2)$ & $(\mathrm{n}=0)$ & $(\mathrm{n}=0)$ & $(\mathrm{n}=2)$ & $(\mathrm{n}=0)$ & $(\mathrm{n}=0)$ \\
Halle & $50.0 \%$ & $0 \%$ & $50.0 \%$ & $0 \%$ & $0 \%$ \\
$(\mathrm{n}=2)$ & $(\mathrm{n}=1)$ & $(\mathrm{n}=0)$ & $(\mathrm{n}=1)$ & $(\mathrm{n}=0)$ & $(\mathrm{n}=0)$ \\
Total & $41.4 \%$ & $27.6 \%$ & $37.9 \%$ & $17.2 \%$ & $10.3 \%$ \\
$(\mathrm{n}=29)$ & $(\mathrm{n}=12)$ & $(\mathrm{n}=8)$ & $(\mathrm{n}=11)$ & $(\mathrm{n}=5)$ & $(\mathrm{n}=3)$ \\
\hline
\end{tabular}

Table 8. Question: "What did you do when asked?". 96.9\% $(\mathrm{n}=31)$ responded. Partly multiple answers.

\begin{tabular}{|c|c|c|c|c|c|c|c|}
\hline City e & $\begin{array}{c}\text { Medical } \\
\text { examination } \\
\text { 1.) }\end{array}$ & $\begin{array}{l}\text { Called } \\
\text { primary } \\
\text { physician } \\
\text { 2.) }\end{array}$ & $\begin{array}{c}\text { Referred } \\
\text { patient to } \\
\text { psychiatrist } \\
\text { 3.) }\end{array}$ & $\begin{array}{c}\text { Without } \\
\text { indication, no } \\
\text { prescription } \\
\text { 4.) }\end{array}$ & $\begin{array}{c}\text { No } \\
\text { prescriptions } \\
\text { for narcotic } \\
\text { drugs 5.) }\end{array}$ & $\begin{array}{l}\text { Prescription } \\
\text { 6.) }\end{array}$ & Other \\
\hline $\begin{array}{l}\text { Munich } \\
(\mathrm{n}=23)\end{array}$ & $\begin{array}{l}13.0 \% \\
(\mathrm{n}=3)\end{array}$ & $\begin{array}{l}26.1 \% \\
(n=6)\end{array}$ & $\begin{array}{l}21.7 \% \\
(\mathrm{n}=5)\end{array}$ & $\begin{array}{c}60.9 \% \\
(\mathrm{n}=14)\end{array}$ & $\begin{array}{c}0 \% \\
(\mathrm{n}=0)\end{array}$ & $\begin{array}{l}30.4 \% \\
(\mathrm{n}=7)\end{array}$ & $\begin{array}{c}4.3 \% \\
(\mathrm{n}=1)\end{array}$ \\
\hline $\begin{array}{l}\text { Frankfurt } \\
\qquad(\mathrm{n}=4)\end{array}$ & $\begin{array}{c}0 \% \\
(\mathrm{n}=0)\end{array}$ & $\begin{array}{c}0 \% \\
(\mathrm{n}=0)\end{array}$ & $\begin{array}{l}25.0 \% \\
(\mathrm{n}=1)\end{array}$ & $\begin{array}{l}75.0 \% \\
(\mathrm{n}=3)\end{array}$ & $\begin{array}{c}0 \% \\
(\mathrm{n}=0)\end{array}$ & $\begin{array}{l}25.0 \% \\
(\mathrm{n}=1)\end{array}$ & $\begin{array}{c}0 \% \\
(\mathrm{n}=0)\end{array}$ \\
\hline $\begin{array}{l}\text { Augsburg } \\
\quad(\mathrm{n}=2)\end{array}$ & $\begin{array}{c}0 \% \\
(\mathrm{n}=0)\end{array}$ & $\begin{array}{c}0 \% \\
(\mathrm{n}=0)\end{array}$ & $\begin{array}{c}0 \% \\
(\mathrm{n}=0)\end{array}$ & $\begin{array}{l}50.0 \% \\
(\mathrm{n}=1)\end{array}$ & $\begin{array}{c}0 \% \\
(\mathrm{n}=0)\end{array}$ & $\begin{array}{l}50.0 \% \\
(\mathrm{n}=1)\end{array}$ & $\begin{array}{c}0 \% \\
(\mathrm{n}=0)\end{array}$ \\
\hline $\begin{array}{l}\text { Halle } \\
(\mathrm{n}=2)\end{array}$ & $\begin{array}{c}0 \% \\
(\mathrm{n}=0)\end{array}$ & $\begin{array}{c}0 \% \\
(\mathrm{n}=0)\end{array}$ & $\begin{array}{l}50.0 \% \\
(\mathrm{n}=1)\end{array}$ & $\begin{array}{l}100 \% \\
(\mathrm{n}=2)\end{array}$ & $\begin{array}{c}0 \% \\
(\mathrm{n}=0)\end{array}$ & $\begin{array}{c}0 \% \\
(\mathrm{n}=0)\end{array}$ & $\begin{array}{c}0 \% \\
(\mathrm{n}=0)\end{array}$ \\
\hline $\begin{array}{c}\text { Total } \\
(\mathrm{n}=31)\end{array}$ & $\begin{array}{c}9.7 \% \\
(n=3)\end{array}$ & $\begin{array}{l}19.4 \% \\
(\mathrm{n}=6)\end{array}$ & $\begin{array}{l}22.6 \% \\
(\mathrm{n}=7)\end{array}$ & $\begin{array}{c}64.5 \% \\
(\mathrm{n}=20)\end{array}$ & $\begin{array}{c}0 \% \\
(\mathrm{n}=0)\end{array}$ & $\begin{array}{l}29.0 \% \\
(\mathrm{n}=9)\end{array}$ & $\begin{array}{c}3.2 \% \\
(\mathrm{n}=1)\end{array}$ \\
\hline
\end{tabular}

1) A medical examination in order to verify whether the request is justified by a medical indication; 2) Called the alleged primary physician to ask whether the patient has a medical indication for methylphenidate; 3) Referred the patient for clarification to a psychiatrist; 4) Stated that I do not issue prescriptions without indication; 5) Stated that I cannot write prescriptions for narcotic drugs; 6) I wrote a prescription for the patient.

A "Rejected as blood donor" (no. 192)

A “Alternative suggestion Vigil (Modafinil)" (no. 223)

\subsubsection{Respondents' Comments}

$20 \%$ of all participants made use of the possibility to comment at the end of the questionnaire. Most comments were written by doctors from Augsburg (27\%). All additional notes made by the doctors and which were not related to a specific question are also evaluated below. Almost $40 \%$ of the doctors thought they were the wrong person for the survey-eg. due to their specialization or due to the fact that they do not prescribe methylphenidate on principle. Further reasons given 
were as follows:

A University: Illegal market by parents whose children take methylphenidate because of an ADHD diagnosis but with a break at the weekend (doctor from Munich)

A Very popular with students-passing exams without Ritalin ${ }^{\circledast}$ virtually impossible

A Nowadays social demands (school, job) are extremely high

A Young people do not want to stop taking Ritalin ${ }^{\circledR}$-despite cardiac side effects-since they experienced an improvement in scholastic achievements

$\Delta$ Ritalin $^{\circledast}$ is prescribed too often and too quickly-in the meantime also for adults

A Germany/USA in comparison with Scandinavian countries: epidemic extents of the ADHD diagnoses (lack of sport, 'Regulative (lifestyle) therapy'2, empathic and normal family situation)

A Doubts about effectiveness of Ritalin ${ }^{\circledR}$ therapy

A More a problem for child and youth psychiatry

A Too often unqualified persons comment on Ritalin $^{\circledR}$-misuse

\subsection{Part 2: Evaluation of World-Wide Literature}

Studies dealing with similar groups of people are regarded as one group in the following. The most important information can be found in a table that lists the literature chronologically by year of publication (cf. Tables 9-14). As mentioned before, not every of those original works was focused exclusively on methylphenidate but on stimulants in general. However, the corresponding table will offer the specific methylphenidate share in relation to the misuse rate of stimulants in general.

\subsubsection{Frequency of Methylphenidate Misuse}

The studies were published in the years 2000 to 2015, and the earliest data collection was in 1992. When looking at students in general (cf. Table 9), lifetime prevalence of methylphenidate misuse varied from $0.8 \%$ [21] to $16.6 \%$ [22]. An average lifetime prevalence of $6.9 \%$ was calculated on the basis of seven studies [21]-[28]. The one-year prevalence ranged from 1.5\% [29] to 7.3\% [30]. An average one-year prevalence of $3.8 \%$ was calculated on the basis of three studies [23] [29] [31].

Additional tables contain information about the prevalence of MPH misuse within the following groups: students in different healthcare professions, school children, civilian population and "Nature"3 readers, patients in an ADHD treatment center and adults with a methylphenidate prescription, adolescents with suspected alcohol and/or drug problems and students/persons with neuroenhancement (cf. Tables 10-14).

\subsubsection{Methylphenidate Misuse and Associated Characteristics}

Three studies found a higher likelihood of MPH misuse among males [32] [33]

${ }^{2}=$ a complex concept of naturopathic therapies

${ }^{3}$ Readers of the natural-science journal "Nature" (www.nature.com) 
Table 9. Studies on "students in general" by country, population examined, author and misuse rate found out. Exclusively original papers.

\begin{tabular}{|c|c|c|c|}
\hline Country & Population examined & Author & Misuse rate \\
\hline USA & $\begin{array}{c}283 \text { students; Massachusetts College of } \\
\text { Liberal Arts; mean age: } 21 \\
\text { (data collection: unknown) }\end{array}$ & $\begin{array}{c}\text { Babcock } \\
\text { and Byrne, } 2000 \\
{[22]}\end{array}$ & $\begin{array}{l}\text { Lifetime } \\
\text { prevalence: } \\
16.6 \%\end{array}$ \\
\hline USA & $\begin{array}{c}150 \text { students; small, competitive college; mean } \\
\text { age: } 20.1 \\
\text { (data collection: unknown) }\end{array}$ & $\begin{array}{c}\text { Low and } \\
\text { Gendaszek, } \\
2002[30]\end{array}$ & $\begin{array}{c}\text { Last-year prevalence: } \\
7.3 \%\end{array}$ \\
\hline USA & $\begin{array}{l}2250 \text { students; University of Michigan; mean } \\
\text { age: } 20.1 \\
\text { (data collection: 2001) }\end{array}$ & $\begin{array}{c}\text { Teter } \\
\text { et al., } 2003[31]\end{array}$ & $\begin{array}{c}\text { Last-year prevalence: } \\
2.5 \%\end{array}$ \\
\hline USA & $\begin{array}{l}4580 \text { students; large mid-western university; } \\
\text { mean age: } 20 \\
\text { (data collection: } 2005 \text { ) }\end{array}$ & $\begin{array}{c}\text { Teter } \\
\text { et al., } 2006 \text { [29] }\end{array}$ & $\begin{array}{c}\text { Last-year prevalence: } \\
1.5 \%\end{array}$ \\
\hline USA & $\begin{array}{l}1025 \text { students; University of New Hampshire } \\
\text { (data collection: 2002) }\end{array}$ & $\begin{array}{l}\text { White } \\
\text { et al., } 2006 \text { [23] }\end{array}$ & $\begin{array}{l}\text { Lifetime prevalence: } \\
16.2 \%\end{array}$ \\
\hline USA & $\begin{array}{l}1253 \text { students; large public university in } \\
\text { mid-Atlantic region; between 17-19 years } \\
\text { (data collection: 2004) }\end{array}$ & $\begin{array}{c}\text { Arria } \\
\text { et al., } 2008[34]\end{array}$ & $\begin{array}{l}\text { Lifetime prevalence: } \\
\qquad 18 \% \\
\text { general stimulants, } \\
\text { of it } 39.6 \% \mathrm{MPH}\end{array}$ \\
\hline USA & $\begin{array}{l}2087 \text { students; currently enrolled in a 2- or } \\
4 \text {-year college + between 18-24 years + US resi- } \\
\text { dent } \\
\text { (data collection: } 2004 \text { ) }\end{array}$ & $\begin{array}{l}\text { DuPont } \\
\text { et al., } 2008 \text { [24] }\end{array}$ & $\begin{array}{l}\text { Lifetime prevalence: } \\
5.3 \%\end{array}$ \\
\hline GER & $\begin{array}{c}1035 \text { pupils }+512 \text { students; mean age: pupils: } \\
\text { 19.3, students: } 24 \\
\text { (data collection: } 2009 / 2010)\end{array}$ & $\begin{array}{c}\text { Franke } \\
\text { et al., } 2011[21]\end{array}$ & $\begin{array}{l}\text { Lifetime prevalence: } \\
\qquad \mathbf{0 . 8 \%} \\
\text { general stimulants, } \\
\text { of it } 100 \% \mathrm{MPH}\end{array}$ \\
\hline GER & $\begin{array}{l}1053 \text { students in Berlin; mean age: } 24.6 \\
\text { (data collection: 2010/2011) }\end{array}$ & $\begin{array}{c}\text { Mache } \\
\text { et al., } 2012 \text { [25] }\end{array}$ & $\begin{array}{l}\text { Lifetime prevalence: } \\
\qquad 2 \%\end{array}$ \\
\hline SUI & $\begin{array}{l}6275 \text { students; University of Zurich/ University } \\
\text { of Basel/ Swiss Federal Institute of Technology } \\
\text { Zurich; mean age: } 23.2 \\
\text { (data collection: 2012/2013) }\end{array}$ & $\begin{array}{c}\text { Maier } \\
\text { et al., } 2013[26]\end{array}$ & $\begin{array}{l}\text { Lifetime prevalence: } \\
\mathbf{5 . 8 \%}\end{array}$ \\
\hline SUI & $\begin{array}{l}1765 \text { students; University of Zurich; median } \\
\text { birth year: } 1986 \\
\text { (data collection: } 2011 \text { ) }\end{array}$ & $\begin{array}{l}\text { Ott and } \\
\text { Biller-Andorno, } \\
2014[27]\end{array}$ & $\begin{array}{l}\text { Lifetime prevalence: } \\
\qquad 5.6 \%\end{array}$ \\
\hline IRI & $\begin{array}{c}1260 \text { students; Rafsanjan University of } \\
\text { Medical Sciences/ Valieasr University/ } \\
\text { Rafsanjan Branch of Islamic Azad University; } \\
\text { mean age: } 21.4 \\
\text { (data collection: } 2008 \text { ) }\end{array}$ & $\begin{array}{l}\text { Rezahosseini et } \\
\text { al., } 2014[35]\end{array}$ & $\begin{array}{l}\text { Half-year prevalence: } \\
\qquad 3.4 \%\end{array}$ \\
\hline NL & $\begin{array}{c}1572 \text { students; nationwide; mean age: } 21.8 \\
\text { (data collection: unknown) }\end{array}$ & $\begin{array}{c}\text { Schelle } \\
\text { et al., } 2015 \text { [28] }\end{array}$ & $\begin{array}{l}\text { Lifetime prevalence: } \\
\qquad 2.5 \%\end{array}$ \\
\hline
\end{tabular}

[35]. $84 \%$ to $74 \%$ of the people with MPH misuse are white [24] [31]. With regard to size of cities and MPH misuse, no correlation was found [21]. A higher lifetime prevalence of MPH misuse was found specifically among people who are 
Table 10. Studies of "Students in different healthcare professions" by country, population examined, author and misuse rate determined. Exclusively original papers.

\begin{tabular}{|c|c|c|c|}
\hline Country & Population examined & Author & Misuse rate \\
\hline \multicolumn{4}{|c|}{ Medical students } \\
\hline IRI & $\begin{array}{l}310 \text { medical students; Tabriz University } \\
\text { of Medical Sciences; mean age: } 21.4 \\
\text { (data collection: 2007) }\end{array}$ & $\begin{array}{l}\text { Habibzadeh et al., } \\
\quad 2011[33]\end{array}$ & $\begin{array}{l}\text { Lifetime prevalence: } \\
\mathbf{8 . 7 \%}\end{array}$ \\
\hline USA & $\begin{array}{l}1,115 \text { medical students; } 4 \text { private and } \\
\text { public medical schools in the greater } \\
\text { Chicago area; mean age: } 25.1 \\
\text { (data collection: } 2011 \text { ) }\end{array}$ & $\begin{array}{l}\text { Emanuel } \\
\text { et al., } 2013 \text { [36] }\end{array}$ & $\begin{array}{l}\text { Lifetime prevalence: } \\
18 \% \text { general stimulants, of } \\
\text { this amount } 41 \% \mathrm{MPH}\end{array}$ \\
\hline & & & $\begin{array}{l}\text { (in this case, rate taken, } \\
\text { not misuse rate!) }\end{array}$ \\
\hline USA & $\begin{array}{l}144 \text { third-year medical students; } \\
\text { southern US medical school; mean age: } 25 \\
\text { (data collection: unknown) }\end{array}$ & $\begin{array}{c}\text { Webb } \\
\text { et al., } 2013[37]\end{array}$ & $\begin{array}{l}\text { Lifetime prevalence: } \\
20 \% \text { general stimulants } \\
\text { (of it } 83 \% \text { specifically for } \\
\text { neuroenhancement), of } \\
\text { this amount } 52 \% \mathrm{MPH}\end{array}$ \\
\hline IR & $\begin{array}{l}229 \text { medical students; Ben Gurion } \\
\text { University of the Negev; mean age: } 26.4 \\
\text { (data collection: } 2013 \text { ) }\end{array}$ & $\begin{array}{l}\text { Cohen } \\
\text { et al., } 2015 \text { [38] }\end{array}$ & $\begin{array}{l}\text { Lifetime prevalence: } \\
\mathbf{8 . 3 \%}\end{array}$ \\
\hline \multicolumn{4}{|c|}{ Doctor of Pharmacy students } \\
\hline USA & $\begin{array}{l}950 \text { Doctor of Pharmacy students; large } \\
\text { urban college of pharmacy in the nor- } \\
\text { theastern region; mean age: } 20 \\
\text { (data collection: 2006) }\end{array}$ & $\begin{array}{c}\text { Lord } \\
\text { et al., } 2009 \text { [39] }\end{array}$ & $\begin{array}{l}\text { Lifetime prevalence: } \\
6.7 \% \text { general stimulants } \\
\text { Last-year prevalence: } 5.0 \% \\
\text { general stimulants, of this } \\
\text { amount } 28 \% \mathrm{MPH}\end{array}$ \\
\hline USA & $\begin{array}{l}407 \text { Doctor of Pharmacy students; } 1 \\
\text { public and } 1 \text { private school of pharmacy in } \\
\text { North Carolina; } 88 \% \text { between } 20-30 \text { years } \\
\text { (data collection: } 2011 / 2012 \text { ) }\end{array}$ & $\begin{array}{c}\text { Volger } \\
\text { et al., } 2014[40]\end{array}$ & $\begin{array}{l}\text { Lifetime prevalence: } \\
1.23 \%\end{array}$ \\
\hline \multicolumn{4}{|c|}{ Dental and dental hygiene students } \\
\hline USA & $\begin{array}{l}243 \text { Dental and dental hygiene students; } \\
\text { dental education institutions in the } \\
\text { south-central region } \\
\text { (data collection: 2008) }\end{array}$ & $\begin{array}{l}\text { McNiel } \\
\text { et al., } 2011[41]\end{array}$ & $\begin{array}{l}\text { With ADHD-diagnosis: } \\
10 \% \text { more than recom- } \\
\text { mended dose, of it } 20 \% \\
\text { with Ritalin-prescription } \\
\text { Without ADHD-diagnosis: } \\
12.4 \% \text { general stimulants, } \\
\text { of this amount } 17 \% \mathrm{MPH}\end{array}$ \\
\hline \multicolumn{4}{|c|}{ Students in healthcare programs } \\
\hline USA & $\begin{array}{l}308 \text { students in healthcare programs } \\
\text { (data collection: unknown) }\end{array}$ & $\begin{array}{l}\text { Herman } \\
\text { et al., } 2011[42]\end{array}$ & $\begin{array}{l}\text { Lifetime prevalence: } 10.4 \% \\
\text { general stimulants, of this } \\
\text { amount } 20 \% \mathrm{MPH}\end{array}$ \\
\hline
\end{tabular}

younger than the age of 24 [23]. Negative results at school/university increased the probability of MPH misuse [21]. 33\% of the patients with ADHD diagnosis had taken more than the recommended dose of methylphenidate at least once [26]. MPH misuse correlated with a higher abuse potential of other substances, such as nicotine, alcohol, marihuana, ecstasy and cocaine [16] [31] [43].

\subsubsection{Factors that Influence Methylphenidate Misuse}

Next to relatives, the peer group is the main influencing factor [25] [33]. 
Table 11. Studies made about "school children" by country, population examined, author and misuse rate determined. Exclusively original papers.

\begin{tabular}{|c|c|c|c|}
\hline Country & Population examined & Author & Misuse rate \\
\hline USA & $\begin{array}{l}\text { 12,237 8th, 10th, and 12th graders } \\
\text { (data collection: 2001) }\end{array}$ & $\begin{array}{c}\text { McCabe } \\
\text { et al., } 2004[43]\end{array}$ & $\begin{array}{l}\text { Last year prevalence: } \\
\qquad \mathbf{4 . 0} \%\end{array}$ \\
\hline GER & $\begin{array}{l}1035 \text { pupils }+512 \text { students; mean age: } \\
\text { school children: } 19.3 \text {, university students: } 24 \\
\text { (data collection: } 2009 / 2010 \text { ) }\end{array}$ & $\begin{array}{c}\text { Franke } \\
\text { et al., } 2011 \text { [21] }\end{array}$ & $\begin{array}{c}\text { Lifetime prevalence: } 1.55 \% \\
\text { general stimulants, of this } \\
\text { number } 94 \% \mathrm{MPH}\end{array}$ \\
\hline SUI & $\begin{array}{l}1139 \text { 10th to 12th graders; Canton of } \\
\text { Zurich;mean age: } 17.1 \\
\text { (data collection: } 2014 \text { ) }\end{array}$ & $\begin{array}{l}\text { Liakoni } \\
\text { et al., } 2015[44]\end{array}$ & $\begin{array}{l}\text { Lifetime prevalence: } 4.0 \% \\
\text { Last-year prevalence: } 2.8 \%\end{array}$ \\
\hline
\end{tabular}

Table 12. Studies of "civilian population" and "“Nature"3readers" by country, population examined, author and misuse rate determined. Exclusively original papers.

\begin{tabular}{|c|c|c|c|}
\hline Country & Population examined & Author & Misuse rate \\
\hline \multicolumn{4}{|c|}{ Civilian population } \\
\hline USA & $\begin{array}{l}\text { Non-institutionalized population; aged } \\
12 \text { or older } \\
\text { (data collection: } 2000-2002)\end{array}$ & $\begin{array}{c}\text { Kroutil } \\
\text { et al., } 2006 \text { [45] }\end{array}$ & $\begin{array}{l}\text { 7.3 million citizens at least } \\
\text { once ADHD-medication, of } \\
\text { it 4,529 thousand MPH }\end{array}$ \\
\hline USA & $\begin{array}{l}\text { 4,297 civilian, non-institutionalized } \\
\text { adults; between } 18-49 \\
\text { (data collection: } 2005 \text { ) }\end{array}$ & $\begin{array}{c}\text { Novak } \\
\text { et al., } 2007 \text { [46] }\end{array}$ & $\begin{array}{c}\text { Lifetime prevalence: } 4.20 \% \\
\text { Last-year prevalence: } \\
0.57 \%\end{array}$ \\
\hline USA & $\begin{array}{l}\text { 24,409 non-institutionalized youths and } \\
\text { adults; between } 16-35 \\
\text { (data collection: } 2003 \text { ) }\end{array}$ & et al., 2007 [32] & $\begin{array}{c}\text { Lifetime prevalence: } \\
10.5 \% \text { men/9.6\% women } \\
\text { general stimulants, of it } \\
78.2 \% \text { men } / 58.4 \% \text { women } \\
\text { MPH }\end{array}$ \\
\hline \multicolumn{4}{|c|}{ "Nature" readers } \\
\hline World-wide & $\begin{array}{l}1.400 \text { "Nature" readers from } \\
60 \text { countries } \\
\text { (data collection: unknown) }\end{array}$ & $\begin{array}{c}\text { Maher, } 2008 \\
{[47]}\end{array}$ & $\begin{array}{l}\text { Lifetime prevalence: } 20 \% \\
\text { general medicaments, of it } \\
62 \% \mathrm{MPH}\end{array}$ \\
\hline
\end{tabular}

\subsubsection{Desired Effects of Methylphenidate}

Depending on the study, the main reason for illicit use was the wish to increase alertness with percentages between $68.9 \%$ to $100 \%$ and is followed by the desire to be able to study better, improve concentration, improve grades and stamina in party situations [23] [35]. Gender had no influence on the reasons mentioned above and $79 \%$ of the consumers used MPH with no qualms [23]. It was regarded as a legitimate action when doctors, pilots and soldiers take drugs in special situations and when bearing great responsibility [48].

\subsubsection{Source of Supply of Methylphenidate}

The most important source of supply (55.7\% to $100 \%)$ were friends and acquaintances [16] [21] [24] [27] [28]. In total, 90\% of the people received methylphenidate free of charge [27]. 58\% of the students who misuse stimulants 
Table 13. Studies of "patients in an ADHD treatment center" and "adults with a methylphenidate prescription" by country, population examined, author and misuse rate determined. Exclusively original papers.

\begin{tabular}{|c|c|c|c|}
\hline Country & Population examined & Author & Misuse rate \\
\hline \multicolumn{4}{|c|}{ Patients in an ADHD treatment center } \\
\hline USA & $\begin{array}{l}545 \text { patients; with/without ADHD- } \\
\text { diagnosis, with short- or long-acting } \\
\text { stimulant medication and/or abuse of } \\
\text { these agents and/or use of illegal or illicit } \\
\text { stimulants; aged } 13 \text { or older; private } \\
\text { ADHD treatment center in a suburb of } \\
\text { Richmond, Virginia } \\
\text { (data collection: unknown) }\end{array}$ & Bright, 2008 [49] & $\begin{array}{c}\text { Lifetime prevalence: } 14.3 \% \\
\text { general stimulants, of this } \\
\text { number } 25.9 \% \mathrm{MPH}\end{array}$ \\
\hline \multicolumn{4}{|c|}{ Adults with prescription for methylphenidate } \\
\hline CAN & $\begin{array}{l}66 \text { adults with prescription for MPH; } \\
\text { aged } 18 \text { or older } \\
\text { (data collection: } 2004 / 2005)\end{array}$ & $\begin{array}{c}\text { Darredeau } \\
\text { et al., } 2007[50]\end{array}$ & $\begin{array}{l}\text { Lifetime prevalence: } \\
29 \%\end{array}$ \\
\hline USA & $\begin{array}{l}42 \text { psychology students with a } \\
\text { prescription for Ritalin or Adderall; } \\
\text { large northeastern university; mean age: } \\
20.7 \text { (data collection: unknown) }\end{array}$ & $\begin{array}{c}\text { Jardin } \\
\text { et al., } 2011 \text { [51] }\end{array}$ & $\begin{array}{c}\text { Lifetime prevalence: } 45 \% \\
\text { general stimulants, of this } \\
\text { number } 31 \% \mathrm{MPH}\end{array}$ \\
\hline
\end{tabular}

Table 14. Studies of "adolescents with suspected alcohol and/or drug problems" and "students/persons with neuroenhancement" by country, population examined, author and misuse rate determined. Exclusively original papers.

\begin{tabular}{|c|c|c|c|}
\hline Country & Population examined & Author & Misuse rate \\
\hline \multicolumn{4}{|c|}{ Adolescents with suspected alcohol and/or drug problems } \\
\hline USA & $\begin{array}{l}231 \text { charts of adolescents with } \\
\text { suspected alcohol and/or drug problem; } \\
\text { mean age: } 15.6 \\
\text { (data collection: charts from 1992-1996) }\end{array}$ & $\begin{array}{l}\text { Marsh } \\
\text { et al., } 2000 \text { [12] }\end{array}$ & $\begin{array}{c}\text { Lifetime prevalence: } \mathbf{1 2 . 8 \%} \\
\text { general stimulants, of this } \\
\text { number } 80 \% \mathrm{MPH}\end{array}$ \\
\hline CAN & $\begin{array}{l}\quad 450 \text { charts of adolescents with } \\
\text { a treatment in a Addiction Center; } \\
\text { mean age: } 15.4 \\
\text { (data collection: charts from 1993-1999) }\end{array}$ & $\begin{array}{l}\text { Williams } \\
\text { et al., } 2004 \text { [52] }\end{array}$ & $\begin{array}{c}\text { Lifetime prevalence: } 20 \% \\
\text { current MPH-addiction: } \\
5.1 \%\end{array}$ \\
\hline \multicolumn{4}{|c|}{ Students/persons with neuroenhancement } \\
\hline GER & $\begin{array}{l}18 \text { students; } 1 . \text { ) use of caffeine and } \\
\text { (psycho-) stimulant drugs for the } \\
\text { purpose of NE 2.) without psychiatric } \\
\text { disorder and without a current } \\
\text { prescription of psychoactive } \\
\text { medication; University of Mainz; mean } \\
\text { age: } 25.8 \text { (data collection: unknown) }\end{array}$ & $\begin{array}{c}\text { Franke } \\
\text { et al., 2012a [48] }\end{array}$ & Lifetime prevalence: $\mathbf{3 8 . 9 \%}$ \\
\hline GER & $\begin{array}{l}20 \text { students; 1.) AMPH or MPH use for } \\
\text { the purpose of NE } 2 . \text { ) without } \\
\text { psychiatric disorder and without a } \\
\text { psychiatric pharmacotherapy } \\
+22 \text { control subjects; University of Mainz } \\
\text { (data collection: unknown) }\end{array}$ & $\begin{array}{c}\text { Franke } \\
\text { et al., 2012b [53] }\end{array}$ & $\begin{array}{l}\text { Lifetime prevalence: } \\
\qquad 55 \%\end{array}$ \\
\hline
\end{tabular}


thought it was easy to obtain them [23].

\subsubsection{Consequences of Methylphenidate Misuse}

91\% of methylphenidate consumers experienced insomnia as a side effect, and many consumers mentioned palpitations, headache, lightheadedness/drowsiness, anxiety as well as flashing, nausea and vomiting [35]. The danger of addiction to Ritalin ${ }^{\circledR}$ was regarded as lower than when taking illegal drugs [48]. Due to their own experience with misuse, $16 \%$ of medical students would prescribe stimulants more thoughtlessly in future [36].

\section{Discussion}

With regard to part 1 and part 2, it is worth noting that there are considerably differences in relation to the population we looked at: In part 1, we surveyed particular German physicians about adult methylphenidate misuse and in part 2 we looked at original works talking about methylphenidate misuse in different populations world-wide. Moreover, in part 2 we included studies even if they viewed at more substances than methylphenidate. The prerequisite was that there were specific data subdivided for the different substances.

\subsection{Part 1: Survey in Germany}

\subsubsection{Response Rates}

The response rate of doctors from Augsburg and Munich was astonishingly high (both 61\%) as was the response rate from Frankfurt (50\%) and Halle (53\%). This relatively high response rate would seem to indicate that this topic is of great interest and relevant. All handwritten notes-eg. very interested in the results, offer to keep in contact also by phone-confirm this observation.

\subsubsection{Patients with Medical Indication for Methylphenidate}

Almost a quarter of the respondents had patients in their care with a medical indication for methylphenidate. The highest prevalence was among doctors in Munich. Due to the relatively small number of participants, this study is not a representative study; however, other studies have already indicated regional differences in the frequency of ADHD diagnoses and MPH prescriptions [54]. According to these studies, ADHD diagnoses in Bavaria in 2011 were $20 \%$ above the German average, and MPH prescriptions were even $24 \%$ above average [54]. By comparison, respondents from Frankfurt and Halle had much lower prevalence with $14 \%$ or rather $12 \%$. These results are confirm data already collected [54]. In Hesse (specifically Frankfurt) and Saxony-Anhalt (specifically Halle an der Saale), ADHD diagnoses and MPH prescriptions were both below national average [54]. In this study, a difference was noted between Munich and Augsburg (both Bavaria). The observation at the federal state level does not explain these differences. In regard to Bavaria, the data at the district level indicate that district levels in particular near Würzburg (Lower Franconia) were the cause of high rates at the federal state level (Reliably ambulatory F90 diagnoses: 10.92/1000 persons; MPH prescriptions: 5.08/1,000 persons) [54]. Between the 
district levels Augsburg and Munich, there were only marginal differences in 2011 [54]. The different size of cities, with a estimated higher substance misuse in big cities, may also be one possible explanation. However, the data of 14,000 German children from 2003 to 2006 showed that-regarding to the size of residence-no significant difference in ADHD prevalence could be found [55].

When doctors had patients in their care with medical indication for methylphenidate, most of these patients were adults. This is quite remarkable as methylphenidate has only been approved for the treatment in adults since 2011 [5]. One respondent (no. 209) complained that too often and too quickly even adults received a prescription. Even against the already mentioned background of the substantial and rapid increase in the number of prescribed methylphenidate for adults, further studies might confirm this hypothesis.

\subsubsection{Inquiries with Regard to Non-Indicated Methylphenidate Prescriptions}

Almost 15\% of the doctors who participated in this survey stated that they had been asked to prescribe methylphenidate without indication. In the four cities mentioned above, the probability of such a non-indicated inquiry (Question 5) was correlated with an additional affirmative answer, whether the same physician had patients in care who had an indication for treatment with MPH (Question 4). Regarding this Munich had the highest correlation. Therefore MPH misuse and ADHD pharmacotherapy could be the two sides of the same coin [22].

Methylphenidate for the purpose of abuse is almost exclusively obtained from friends and acquaintances [16] [21] [24] [27] [28]. Against this background and knowing from the comparison with the literature that only a small percentage of this drug is prescribed by a doctor, the prevalence discovered in this paper (15\%) would seem to be quite high. It must be pointed out that a request and a non-indicated prescription does not automatically go together. As reported in the results, most doctors (65\%) rejected those requests. However, the estimated number of prescriptions may be marginally increased, supposing that the case of one respondent (no. 1), who admitted that it was likely that he prescribed methylphenidate without medical indication, but did not recognize it as non-indicated in the situation, is not an isolated case. The results support the assumption that Germans try to obtain the drug from a doctor, but it is fortunately no promising tract.

The questionnaire was also sent to doctors with a specialization in internal medicine. The assumption was that patients may go from one doctor to another in an attempt to obtain methylphenidate from a different source. Numerous notes on the inquiry sheets, however, suggest specialists do not run into this. About half of the doctors who already were asked for methylphenidate without indication also care for patients in which administration was indicated. With this knowledge, a separate survey among doctors with several "methylphenidate patients" in their patient base, would seem to be important. The frequency at which doctors were confronted with non-indicated requests appears to be rather low. 
The doctors who participated in this survey were also asked whether adults wanted the drug for themselves or for a child. The result was that $80 \%$ of the patients wanted the medicine for themselves, more than $80 \%$ of these patients were between 20 and 40 years old. Given the fact that examinations and time pressure among students and young working people are seen as a stress factor, these results are not surprising [15]. The ages between 20 and 40 discovered in this survey also correlates with the age determined in the examined studies on misuse [23] [31]. Against this background, some of the respondents' notes are very interesting. One doctor (no. 136) thinks that nowadays social requirements of school and jobs are extremely high. Another doctor (no. 47) mentioned the popular intake by students because passing state examinations without Ritalin ${ }^{\circledR}$ is virtually impossible. A cardiologist (no. 68) emphasized that methylphenidate often has cardiac side effects. Nevertheless, young people are unwilling to stop the taking the drug because they want to improve school performance ("Frequent cardiac side effects due to Ritalin-therapy (tachycardia), but they still don't want to stop taking it because of alleged higher grades"). Due to the numerous and partly serious side effects which were described in the introduction, it is necessary to monitor the patient before and during the phamacotherapy [8].

Only $10 \%$ of the adults with an indicated request had been examined by doctors. It is not clear whether a doctor did not consider or whether the patient refused an examination. It is easy to imagine that a patient suffering from drug misuse strictly refuses an examination. Further studies should deal with this aspect.

The information that an illegal market for methylphenidate exists at the University of Munich is quite alarming. One respondent (no. 13) mentioned the fact that parents of children with ADHD diagnosis do not give their children methylphenidate over the weekend but sell this "saved" drug at the university. To date studies about MPH misuse have indicated an illegal market at schools and universities; however, only university students and school children giving away or selling methylphenidate have been mentioned in these studies [16] [24] [36] [37] [46] [56]. The fact that parents knowingly deprive their children of the drug-which is funded by the health insurance schemes-was not mentioned in any publication. As a consequence, doctors should not only ensure good compliance on the part of the child, but also take into account the danger of interruptions in treatment induced by the parent. The consequences of such interruptions for children-whether in the long or short term-are unknown. The welcome effects of Ritalin ${ }^{\circledR}$ only last for six hours [1]. Possibly numerous and short breaks in therapy are responsible for an increase in typical ADHD symptoms. These symptoms could be misinterpreted by doctors and result in higher doses.

The note of a transfusion physician (no. 192) underlines the fact that methylphenidate is a medicine that is not to be underestimated. According to this physician, patients who use methylphenidate are definitely not acceptable as blood donors. Following oral application of an immediate-release preparation, the 
half-life period in plasma is two to three hours and this value doubles after application of a retard preparation [1] [57] [58]. Thus, drug residues in the blood bag cannot be excluded. According to a transfusion physician of the "Bavarian Red Cross (BRK)", there were no official guidelines about methylphenidate in particular which were based on studies [59]. Blood donor services have come to internal agreements and followed guidelines written by the Medical Association in cooperation with the German Paul Ehrlich Institute [59]. With regard to donors with medication intake, the Medical Association demands in an upgraded version of 2010 that they will be postponed for an estimated time [60]. The provision of each medicine depends on the pharmacokinetic and indication [60]. The result is that all patients with psychostimulant therapy-which include methylphenidate-are excluded from donating blood [59]. The reason for exclusion is a matter of concern with regard to possible paradox reactions on the recipient's side [59]. Obviously, this quality requirement regarding stored blood can only be realized in actual practice if there is a relationship of trust between doctors and donors. In the preliminary talk, the donor fails to disclose the use of methylphenidate. But if a blood donor misuses methylphenidate, inhibition level will probably be quite high, and the individual will conceal that he/she takes methylphenidate more frequently.

According to a physician in the Department of Addiction Medicine (no. 76), methylphenidate abuse among his patients had decreased in recent years. $\mathrm{He}$ doubted that the importance of methylphenidate had declined, however. His assumption was that more and more "crystal meth", a methamphetamine, is being consumed. He stated that it was cheaper and much easier to obtain. "Crystal meth" is a substance similar to methylphenidate and causes similar effects [4]. Data released by the police about the development of drug abuse confirm an increasing spread of amphetamines and methamphetamines [61].

In Germany, the trend to obtain the entrance examination requirement for higher education has become very popular in recent years [62]. 57\% of the school leavers completed their secondary education with the requirements for higher education [62]. Obviously, more and more people think that an academic qualification is a "must" to obtain a safe job. Justifications for the improper MPH use illustrate that school children take methylphenidate in order to reach this aim. The reasons mentioned most frequently are as follows: increasing level of attention and concentration, enhancing learning behavior and grades [23] [35]. It would seem likely that there is a connection between a change of school and the highest frequency of ADHD diagnoses around the age of 10 [54]. Therefore, MPH abuse can partly be seen as a consequence of today's achievement-orientated society.

The number of ADHD diagnoses and MPH prescriptions has been increasing considerably [7] [54] [63]. If the misuse of stimulants is not considered as a separate problem, but as part of the misuse problem of stimulants used to treat ADHD then today's ADHD therapies should be looked at very critically [23]. Particularly with regard to the updated license for methylphenidate in 2011 with 
an approval for treatment of adults, a skeptical discussion seems to be of special significance [5]. The possibility of misuse should be taken into account when prescribing methylphenidate [22].

\subsection{Part 2: Evaluation of Literature World-Wide}

\subsubsection{Frequency of Methylphenidate Misuse}

When comparing the different studies already published, it is quite remarkable that only four of the 34 studies deal with German data, and the majority of studies $(n=20)$ were conducted in the USA. Moreover, it was primarily university students who were linked to methylphenidate misuse, and, apart from the DAK-health report, virtually no data exists about the working people [7]. It is obvious that-despite the numerous reports in the media-additional data is needed not only about Germany but also about different occupational groups.

Essential differences among students became clear: lifetime prevalence varied from $0.8 \%$ [21] to $16.6 \%$ [22]. The reason for this could be the different methods of conducting studies. In other words, Franke et al. recognized deviating prevalence in a survey among surgeons on the issue of the neuroenhancement with prescription drugs and illicit drugs depending on the method of questioning [64]. When using an anonymous questionnaire, the life-time prevalence was $9 \%$ and when using the random-response-technique (RRT) among the same cohort the prevalence was $20 \%$ [64]. Compared to common questioning methods, the RRT provide more reliable data, especially in cases of indiscreet and incriminating questions [65]. None of the studies examined in the present paper used this technique. Hence, it is assumed that there is a high number of unrecorded methylphenidate misuse cases. This fact is underlined by information on differences between data of own misuse and alleged misuse by fellow students [40].

\subsubsection{Methylphenidate Misuse and Associated Characteristics}

The methylphenidate misuse seems to be highly associated with the male gender and a Caucasian origin [24] [30] [31] [32] [33] [35]. Furthermore, young adults around the age of 20 as well as college students present themselves as particularly at risk [23] [31]. With this knowledge it is possible to narrow down a group of persons that should be given attention and targeted by preventive measures. It is not surprising that $89 \%$ of the Israeli medical students who misuse MPH began misuse at university, because neuroenhancement is more frequently reported by students with higher levels of performance pressure and if the pressure is considered as more burdensome by those students [28] [38] [44] [53]. One can imagine that performance pressure is very common among medical students. This point gain even more significance when knowing that stimulant taking-whether coffee or Ritalin ${ }^{\circledR}$-is regarded partly only as a way of achieving aims such as good marks [48]. However, they feel better if the results were achieved without enhancers [48].

Misuse is most likely not more widespread in large cities, as there was, unexpectedly, no difference detectable in regard to the size of cities [21].

There was a strong association between MPH misuse and ADHD diagnoses. It 
appears that persons with a MPH prescription are given to misuse methylphenidate more often [26] [50] [51]. Perhaps this increased likelihood persists after the end of therapy. Half of the university students with a stimulant prescription in the past, were still taking the drug for the purpose of neuroenhancing at the time of the survey [42]. Based on this awareness, a potential misuse after a pharmacological therapy in patients with ADHD should be considered.

It is assumed that a pharmacological therapy with the psychostimulant methylphenidate-above all because of certain similarity to amphetamine and cocaine-could function as a gateway drug [32]. Looking at the high correlation between MPH misuse and misuse of other substances such as marijuana, ecstasy and cocaine, the hypothesis does not appear to be unfounded [16] [31] [43]. It should be noted that an influence in the opposite direction is also conceivable, so that persons with experience of abuse are more likely to use methylphenidate [66].

\subsubsection{Factors that Influence Methylphenidate Misuse}

The peer group turned out to be the most important influencing factor [33]. Typically, a peer group has a leader with a strong and accepted influence, resulting in the high conformity of the group [67]. A study with a view to students with pharmacological neuroenhancement showed the influence of the peer group and also a division concerning the transfer of information. On the one hand, there is a well-informed group which supports or at least tolerates neuroenhancement and partly practices it themselves; on the other hand, there are students who are skeptical about the topic [68]. The result is two information systems that are completely independent of each other wherein students who take NE avoid critical discussions [68]. Kwan et al. were able to show in their study on the subject of the influence of a substance abusing peer leader on group members, who are vulnerable to misuse, that the risk in this situation declines for women and increases for men [69]. Moreover, this is a revealing point since the majority of the abusing students are male [32] [33] [35]. These findings are of central importance to initiate education programs as far as possible within the peer group and even gender-specific programs can possibly be beneficial.

\subsubsection{Desired Effects of Methylphenidate}

The fact that the taking of a stimulant by physicians, pilots or soldiers is viewed in certain situations and at a high level of responsibility as legitimate, may have alarming consequences on the professional environment [48]. The issue of doping in sports is strictly regulated by the World Anti-Doping Agency (WADA), and methylphenidate is on the list of prohibited substances [70]. At a major computer fair (Gamescom) with contest prizes of a million Euros and more, drug testing was carried out for first time in 2015 for prescription drugs (Rita$\operatorname{lin}^{\oplus}$, Adderall ${ }^{\circ}$ ) [71]. Nevertheless, there is no equivalent regulation in the working world. When considering soldiers, surgeons or brokers, the question arises of whether "doping" should be accepted-unlike in the case of sports-and what acceptance says about a society. These are not speculations about the future. US 
army flight surgeons are already obligated indirectly to take dexedrine during missions [72].

One third of the students who misuse general stimulants are afraid of "brain dopers" [25]. Mental performance will become even more important to future generations. Out of the concern that classmates might be being given methylphenidate by their parents, it is possible that some parents will feel compelled to give methylphenidate to their own children in order to give them the best opportunities [47].

\subsubsection{Source of Supply of Methylphenidate}

Methylphenidate is primarily obtained from friends and acquaintances [16] [21] [24] [27] [28]. They usually have a valid prescription, therefore greater efforts are important to raise patient compliance [16] [46]. In the case of stimulant therapies, treating physicians bear a great responsibility with regard to weighing use and risks and should be aware of possible overdose and/or diversion [29] [34]. Special care and strict controls must be taken for patients with illicit substance use in the past [50]. Furthermore, physicians should bear in mind that patients may simulate ADHD symptoms in order to receive a stimulant prescription [24]. Physicians need additional training to be able to identify those patients [46]. Novak et al. also argue for additional monitoring programs on prescription drugs or strict penalties for lax prescribing practices [46]. More generally, wide-ranging prevention measures-which involve parents and fellow students-are required [34]. Early programs focusing on middle-school children and thus before the usual starting age, would be beneficial [32]. De Santis et al. note that it gets exponentially heavier to get in touch with students due to increasing widespread stimulant misuse (20-30\% at most of the US universities) [73]. Mainly in regard to the aspect of financial constraints, it would be more promising to look for the small share of "distributors" (2-4\% at most of the US universities) [73].

\subsubsection{Consequences of Methylphenidate Misuse}

Emanuel et al. detected common psychostimulant misuse among medical students and argued for further studies on side effects, clinical implications and the intake during the years as an assistant doctor [36]. Nearly one fifth of medical students would prescribe stimulants more thoughtlessly to their own patients in future; consequently, further scientific consideration is needed [36]. Data on widespread misuse among surgeons, collected by Franke et al., need closer examination [64]. Although certain students assume that an intake in special situations, eg. as by a surgeon, is legitimate, critical analyses with regard to the risks posed by intake are lacking [48]. It is to be considered that a surgeon operating under the effect of drugs overestimates his or her capabilities, this can quickly pose an increased risk to patients [64].

\section{Study Limitations}

The aim of the present paper was to conduct a pilot study. Hence, weaknesses in 
the study design were tolerated. In particular, the small case number, the not validated questionnaire, and the fact that the results are not based on statistical analysis but exclusively on descriptive statistics must be mentioned. Moreover, responses of the questionnaire may contain subjective assessments of the participants. The decision on participation was made by the participants themselves, which may bias the validity of the results as well. Due to these methodology deficiencies, the limited informative value must be considered. Furthermore it must be remembered that the studies of the comparison with the literature are structured differently and are coming from different populations, which is impeding the comparison with our results coming from the physicians about adult misuse.

\section{Conclusion}

The survey of primary care physicians/internists as well as the analysis of world-wide studies indicates that the issue of methylphenidate misuse is a major problem and cannot be limited to the discipline of psychiatry. On the contrary, it affects other medical fields. MPH-prescriptions for children should also consider the option that adults and former patients with an intake of methylphenidate may misuse the substance. This has not yet been adequately investigated.

\section{Declaration of Interest}

The authors report no conflicts of interest.

\section{References}

[1] Yacoub, Krause, K.H., Dresel, S. and Krause, J. (2001) Wirkmechanismus von Methylphenidat. In: Voss Hv, Ed., Kinderärztliche Praxis; Sonderheft "Unaufmerksam und hyperaktiv", Kirchheim-Verlag, Mainz, 23-27.

[2] Fünfte Verordnung über die den Betäubungsmitteln gleichgestellten Stoffe (Fünfte Betäubungsmittel-Gleichstellungsverordnung-5. BtMGlV, vom 6, April 1971.

[3] Kanner, B.I. and Schuldiner, S. (1987) Mechanism of Transport and Storage of Neurotransmitters. Critical Reviews in Biochemistry, 22, 1-38. https://doi.org/10.3109/10409238709082546

[4] Han, D.D. and Gu, H.H. (2006) Comparison of the Monoamine Transporters from Human and Mouse in Their Sensitivities to Psychostimulant Drugs. BMC Pharmacology, 6, 6. https://doi.org/10.1186/1471-2210-6-6

[5] Gemeinsamer Bundesausschuss. Arzneimittel-Richtlinie/Anlage III Nummer 44 (Stimulantien). Beschlussdatum: 23 June 2011. https://www.g-ba.de/downloads/40-268-1684/2011-06-23_AM-RL-III_Nr44-Stimul antien_TrG.pdf

[6] AOK. Arzneimittelrabattverträge der AOK. Tender XII-XIV. Juni 2015. https://www.aok-gesundheitspartner.de/imperia/md/gpp/bund/arzneimittel/rabatt/ aok_rv_vertragsuebersicht_xii_xiv_juni2015.pdf

[7] IGES-InstitutfürGesundheits- und Sozialforschung (2015) DAK-Gesundheitsreport 2015. IGES Institut GmbH, Berlin.

[8] Barkley, R.A., McMurray, M.B., Edelbrock, C.S. and Robbins, K. (1990) Side Effects of Methylphenidate in Children with Attention Deficit Hyperactivity Disorder: A 
Systemic, Placebo-Controlled Evaluation. Pediatrics, 86, 184-192.

[9] Kelly, K.L., Rapport, M.D. and DuPaul, G.J. (1988) Attention Deficit Disorder and Methylphenidate: A Multi-Step Analysis of Dose-Response Effects on Children's Cardiovascular Functioning. International Clinical Psychopharmacology, 3, 167181. https://doi.org/10.1097/00004850-198804000-00007

[10] Klein-Schwartz, W. (2002) Abuse and Toxicity of Methylphenidate. Current Opinion in Pediatrics, 14, 219-223. https://doi.org/10.1097/00008480-200204000-00013

[11] Konrad-Bindl, D.S., Gresser, U. and Richartz, B.M. (2016) Changes in Behavior as Side Effects in Methylphenidate Treatment: Review of the Literature. Neuropsychiatric Disease and Treatment, 12, 2635-2647.

https://doi.org/10.2147/NDT.S114185

[12] Marsh, L.D., Key, J.D. and Payne, T.P. (2000) Methylphenidate Misuse in Substance Abusing Adolescents. Journal of Child \& Adolescent Substance Abuse, 9, 1-14. https://doi.org/10.1300/J029v09n03_01

[13] Forlini, C., Schildmann, J., Roser, P., Beranek, R. and Vollmann, J. (2014) Knowledge, Experiences and Views of German University Students toward Neuroenhancement: An Empirical-Ethical Analysis. Neuroethics, 8, 83-92. https://doi.org/10.1007/s12152-014-9218-Z

[14] Frauger, E., Amaslidou, D., Spadari, M., Allaria-Lapierre, V., Braunstein, D., Sciortino, V., Thirion, X., Djezzar, S. and Micallef, J. (2016) Patterns of Methylphenidate Use and Assessment of Its Abuse among the General Population and Individuals with Drug Dependence. European Addiction Research, 22, 119-126.

[15] Techniker Krankenkasse. TK-Stress-Studie NRW Studenten. Ergebnisseeinerrepräsentativen Forsa-Umfrageaus Mai 2012.

https://www.tk.de/centaurus/servlet/contentblob/456454/Datei/80754/Forsa-Studie \%20Studentenalltag\%20in\%20NRW.pdf

[16] Barrett, S.P., Darredeau, C., Bordy, L.E. and Pihl, R.O. (2005) Characteristics of Methylphenidate Misuse in a University Student Sample. The Canadian Journal of Psychiatry, 50, 457-461. https://doi.org/10.1177/070674370505000805

[17] Wilens, T.E., Adler, L.A., Adams, J., Sgambati, S., Rotrosen, J., Sawtelle, R., Utzinger, L. and Fusillo, S. (2008) Misuse and Diversion of Stimulants Prescribed for ADHD: A Systematic Review of the Literature. Journal of the American Academy of Child \& Adolescent Psychiatry, 47, 21-31. https://doi.org/10.1097/chi.0b013e31815a56f1

[18] KV Bayern. Honorarsystematik ab 01.01.2015 (HVM), Kalkulatorische Jahresfallwerte 2015, Fallwerte, Fallzahlen und Quoten. 2015 https://www.kvb.de/abrechnung/honorar/honorar-ab-010115/

[19] KV Sachsen-Anhalt. Offizielles Mitteilungsblatt der Kassenärztlichen Vereinigung Sachsen-Anhalt. Fallwerte 4.Quartal 2015. 2015. http://www.kvsa.de/fileadmin/user_upload/PDF/Publikationen/PRO_Magazine/PR O_2015/PRO_10-2015_Beilage_AGR_4-2015.pdf

[20] Braunstein, D. (2016) Employee of the Association of Statutory Health Insurance Physicians (Hesse). Expert Discussion, Telephone call on 25 May 2016.

[21] Franke, A.G., Bonertz, C., Christmann, M., Huss, M., Fellgiebel, A., Hildt, E. and Lieb, K. (2011) Non-Medical Use of Prescription Stimulants and Illicit Use of Stimulants for Cognitive Enhancement in Pupils and Students in Germany. Pharmacopsychiatry, 44, 60-66. https://doi.org/10.1055/s-0030-1268417

[22] Babcock, Q. and Byrne, T. (2000) Student Perceptions of Methylphenidate Abuse at a Public Liberal Arts College. Journal of American College Health, 49, 143-145. 
https://doi.org/10.1080/07448480009596296

[23] White, B.P., Becker-Blease, K.A. and Grace-Bishop, K. (2006) Stimulant Medication Use, Misuse, and Abuse in an Undergraduate and Graduate Student Sample. Journal of American College Health, 54, 261-268. https://doi.org/10.3200/JACH.54.5.261-268

[24] DuPont, R.L., Coleman, J.J., Bucher, R.H. and Wilford, B.B. (2008) Characteristics and Motives of College Students Who Engage in Nonmedical Use of Methylphenidate. The American Journal on Addictions, 17, 167-171. https://doi.org/10.1080/10550490802019642

[25] Mache, S., Eickenhorst, P., Vitzthum, K., Klapp, B.F. and Groneberg, D.A. (2012) Cognitive-Enhancing Substance Use at German Universities: Frequency, Reasons and Gender Differences. Wiener Medizinische Wochenschrift, 162, 262-271. https://doi.org/10.1007/s10354-012-0115-y

[26] Maier, L.J., Liechti, M.E., Herzig, F. and Schaub, M.P. (2013) To Dope or Not to Dope: Neuroenhancement with Prescription Drugs and Drugs of Abuse among Swiss University Students. PLoS ONE, 8, e77967. https://doi.org/10.1371/journal.pone.0077967

[27] Ott, R. and Biller-Andorno, N. (2014) Neuroenhancement among Swiss StudentsA Comparison of Users and Non-Users. Pharmacopsychiatry, 47, 22-28.

[28] Schelle, K.J., Olthof, B.M., Reintjes, W., Bundt, C., Gusman-Vermeer, J. and van Mil, A.C. (2015) A Survey of Substance Use for Cognitive Enhancement by University Students in the Netherlands. Frontiers in Systems Neuroscience, 9, 10. https://doi.org/10.3389/fnsys.2015.00010

[29] Teter, C.J., McCabe, S.E., LaGrange, K., Cranford, J.A. and Boyd, C.J. (2006) Illicit Use of Specific Prescription Stimulants among College Students: Prevalence, Motives, and Routes of Administration. Pharmacotherapy, 26, 1501-1510. https://doi.org/10.1592/phco.26.10.1501

[30] Low, K.G. and Gendaszek, A.E. (2002) Illicit Use of Psychostimulants among College Students: A Preliminary Study. Psychology Health \& Medicine, 7, 283-287. https://doi.org/10.1080/13548500220139386

[31] Teter, C.J., McCabe, S.E., Boyd, C.J. and Guthrie, S.K. (2003) Illicit Methylphenidate Use in an Undergraduate Student Sample: Prevalence and Risk Factors. Pharmacotherapy, 23, 609-617.

[32] Wu, L.T., Pilowsky, D.J., Schlenger, W.E. and Galvin, D.M. (2007) Misuse of Methamphetamine and Prescription Stimulants among Youths and Young Adults in the Community. Drug and Alcohol Dependence, 89, 195-105. https://doi.org/10.1016/j.drugalcdep.2006.12.020

[33] Habibzadeh, A., Alizadeh, M., Malek, A., Maghbooli, L., Shoja, M.M. and Ghabili, K. (2011) Illicit Methylphenidate Use among Iranian Medical Students. Prevalence and Knowledge . Drug Design, Development and Therapy, 5, 71-576.

[34] Arria, A.M., Caldeira, K.M., O’Grady, K.E., Vincent, K.B., Johnson, E.P. and Wish, E.D. (2008) Nonmedical Use of Prescription Stimulants among College Students: Associations with Attention-Deficit-Hyperactivity Disorder and Polydrug Use. Pharmacotherapy, 28, 156-169. https://doi.org/10.1592/phco.28.2.156

[35] Rezahosseini, O., Roohbakhsh, A., Tavakolian, V. and Assar, S. (2014) Drug Abuse among University Students of Rafsanjan, Iran. Iranian Journal of Psychiatry and Behavioral Sciences, 8, 81-85.

[36] Emanuel, R.M., Frellsen, S.L., Kashima, K.J., Sanguino, S.M., Sierles, F.S. and Lazarus, C.J. (2013) Cognitive Enhancement Drug Use among Future Physicians: Find- 
ings from a Multi-Institutional Census of Medical Students. Journal of General Internal Medicine, 28, 1028-1034. https://doi.org/10.1007/s11606-012-2249-4

[37] Webb, J.R., Valasek, M.A. and North, C.S. (2013) Prevalence of Stimulant Use in a Sample of US Medical Students. Annals of Clinical Psychiatry, 25, 27-32.

[38] Cohen, Y.G., Segev, R.W., Shlafman, N., Novack, V. and Ifergane, G. (2015) Methylphenidate Use among Medical Students at Ben-Gurion University of the Negev. Journal of Neurosciences in Rural Practice, 6, 320-325. https://doi.org/10.4103/0976-3147.158749

[39] Lord, S., Downs, G., Furtaw, P., Chaudhuri, A., Silverstein, A., Gammaitoni, A. and Budman, S. (2009) Nonmedical Use of Prescription Opioids and Stimulants among Student Pharmacists. Journal of the American Pharmacists Association, 49, 519528. https://doi.org/10.1331/JAPhA.2009.08027

[40] Volger, E.J., McLendon, A.N., Fuller, S.H. and Herring, C.T. (2014) Prevalence of Self-Reported Nonmedical Use of Prescription Stimulants in North Carolina Doctor of Pharmacy Students. Journal of Pharmacy Practice, 27, 158-168. https://doi.org/10.1177/0897190013508139

[41] McNiel, A.D., Muzzin, K.B., DeWald, J.P., McCann, A.L. and Schneiderman, E.D. (2011) The Nonmedical Use of Prescription Stimulants among Dental and Dental Hygiene Students. Journal of Dental Education, 75, 365-376.

[42] Herman, L., Shtayermman, O., Aksnes, B., Anzalone, M., Cormerais, A. and Liodice, C. (2011) The Use of Prescription Stimulants to Enhance Academic Performance among College Students in Health Care Programs. The Journal of Physician Assistant Education, 22, 15-22. https://doi.org/10.1097/01367895-201122040-00003

[43] McCabe, S.E., Teter, C.J., Boyd, C.J. and Guthrie, S.K. (2004) Prevalence and Correlates of Illicit Methylphenidate Use among 8th, 10th, and 12th Grade Students in the United States, 2001. Journal of Adolescent Health, 35, 501-504. https://doi.org/10.1016/S1054-139X(04)00067-9

[44] Liakoni, E., Schaub, M.P., Maier, L.J., Glauser, G.V. and Liechti, M.E. (2015) The Use of Prescription Drugs, Recreational Drugs, and "Soft Enhancers" for Cognitive Enhancement among Swiss Secondary School Students. PLOS ONE, 10, e014128. https://doi.org/10.1371/journal.pone.0141289

[45] Kroutil, L.A., Van Brunt, D.L., Herman-Stahl, M.A., Heller, D.C., Bray, R.M. and Penne, M.A. (2006) Nonmedical Use of Prescription Stimulants in the United States. Drug and Alcohol Dependence, 84, 135-143. https://doi.org/10.1016/j.drugalcdep.2005.12.011

[46] Novak, S.P., Kroutil, L.A., Williams, R.L. and Van Brunt, D.L. (2007) The Nonmedical Use of Prescription ADHD Medications: Results from a National Internet Panel. Substance Abuse Treatment, Prevention, and Policy, 2, 32. https://doi.org/10.1186/1747-597X-2-32

[47] Maher, B. (2008) Poll Results: Look Who's Doping. Nature, 452, 674-675. https://doi.org/10.1038/452674a

[48] Franke, A.G., Lieb, K. and Hildt, E. (2012) What Users Think about the Differences between Caffeine and Illicit/Prescription Stimulants for Cognitive Enhancement. PLOS ONE, 7, e40047. https://doi.org/10.1371/journal.pone.0040047

[49] Bright, G.M. (2008) Abuse of Medications Employed for the Treatment of ADHD: Results from a Large-Scale Community Survey. The Medscape Journal of Medicine, $10,111$.

[50] Darredeau, C., Barrett, S.P., Jardin, B. and Pihl, R.O. (2007) Patterns and Predictors of Medication Compliance, Diversion, and Misuse in Adult Prescribed Methylphe- 
nidate Users. Human Psychopharmacology, 22, 529-536.

https://doi.org/10.1002/hup.883

[51] Jardin, B., Looby, A. and Earleywine, M. (2011) Characteristics of College Students with Attention-Deficit Hyperactivity Disorder Symptoms Who Misuse Their Medications. Journal of American College Health, 59, 373-377.

[52] Williams, R.J., Goodale, L.A., Shay-Fiddler, M.A., Gloster, S.P. and Chang, S.Y. (2004) Methylphenidate and Dextroamphetamine Abuse in Substance-Abusing Adolescents. The American Journal on Addictions, 13, 381-389. https://doi.org/10.1080/10550490490483053

[53] Franke, A.G., Schwarze, C.E., Christmann, M., Bonertz, C., Hildt, E. and Lieb, K. (2012) [Characteristics of University Students Using Stimulants for Cognitive Enhancement: A Pilot Study]. Psychiatrische Praxis, 39, 174-180.

https://doi.org/10.1055/s-0031-1298900

[54] Grobe, T.G., Bitzer, E.M. and Schwartz, F.W. (2013) BARMERGEK Arztreport 2013. Schwerpunkt: Aufmerksamkeitsdefizit-/Hyperaktivitätsstörungen ADHS. Berlin.

[55] Schlack, R., Hölling, H., Kurth, B.M. and Huss, M. (2007) Die Prävalenz der Aufmerksamkeitsdefizit-/Hyperaktivitätsstörung (ADHS) bei Kindern und Jugendlichen in Deutschland: Erste Ergebnisse aus dem Kinder- und Jugendgesundheitssurvey (KiGGS). Bundesgesundheitsblatt-Gesundheitsforschung-Gesundheitsschutz, 50, 827-835. https://doi.org/10.1007/s00103-007-0246-2

[56] Chen, L.Y., Crum, R.M., Strain, E.C., Alexander, G.C., Kaufmann, C. and Mojtabai, R. (2016) Prescriptions, Nonmedical Use, and Emergency Department Visits Involving Prescription Stimulants. The Journal of Clinical Psychiatry, 77, e297-e304. https://doi.org/10.4088/JCP.14m09291

[57] Srinivas, N.R., Hubbard, J.W., Quinn, D. and Midha, K.K. (1992) Enantioselective Pharmacokinetics and Pharmacodynamics of $\mathrm{dl}$-threo-Methylphenidate in Children with Attention Deficit Hyperactivity Disorder. Clinical Pharmacology \& Therapeutics, 52, 561-568. https://doi.org/10.1038/clpt.1992.185

[58] Markowitz, J.S., Straughn, A.B., Patrick, K.S., DeVane, C.L., Pestreich, L., Lee, J., Wang, Y. and Muniz, R. (2003) Pharmacokinetics of Methylphenidate after Oral Administration of Two Modified-Release Formulations in Healthy Adults. Clinical Pharmacokinetics, 42, 393-401. https://doi.org/10.2165/00003088-200342040-00007

[59] Eicke, H. (2015) Medical Specialist in Transfusion Medicine, Deputy Head of Control of the Bavarian Red Cross. Expert Discussion, Telephone call on 30 October 2015.

[60] Bundesärztekammer, Ed., RichtlinienzurGewinnung von Blut und Blutbestandteilen und zurAnwendung von Blutprodukten (Hämotherapie). Aufgestelltgemäß $\$ \$ 12 \mathrm{a}$ u. 18 Transfusionsgesetz von der BundesärztekammerimEinvernehmenmitdem Paul-Ehrlich-Institut. Gesamtnovelle 2005, mitRichtlinienanpassung 2010. Deutscher Ärzte-Verlag. 2010.

http://www.bundesaerztekammer.de/fileadmin/user_upload/downloads/RiliHaemo therapie2010.pdf

[61] Bundeskriminalamt (2014) PolizeilicheKriminalstatistik. Bundesrepublik Deutschland. Jahrbuch 2014. 62. Auflage. Wiesbaden.

[62] Autorengruppe Bildungsberichterstattung (2014) Bildung in Deutschland 2014. Einindikatorengestützter Berichtmiteiner Analysezur Bildung von Menschen mit Behinderungen. W. Bertelsmann Verlag GmbH \& Co. KG, Bielefeld.

[63] Grobe, T., Steinmann, S., AQUA-Institut für angewandte Qualitätsförderung (2015) Gesundheitsreport 2015. Techniker Krankenkasse, Hamburg. 
[64] Franke, A.G., Bagusat, C., Dietz, P., Hoffmann, I., Simon, P., Ulrich, R. and Lieb, K. (2013) Use of Illicit and Prescription Drugs for Cognitive or Mood Enhancement among Surgeons. BMC Medicine, 11, 102. https://doi.org/10.1186/1741-7015-11-102

[65] Warner, S.L. (1965) Randomized Response: A Survey Technique for Eliminating Evasive Answer Bias. Journal of the American Statistical Association, 60, 63-66. https://doi.org/10.1080/01621459.1965.10480775

[66] Sweeney, C.T., Sembower, M.A., Ertischek, M.D., Shiffman, S. and Schnoll, S.H. (2013) Nonmedical Use of Prescription ADHD Stimulants and Preexisting Patterns of Drug Abuse. Journal of Addictive Diseases, 32, 1-10. https://doi.org/10.1080/10550887.2012.759858

[67] Springer Gabler Verlag, Ed., Gabler Wirtschaftslexikon, Stichwort: Peer Group. Version 8. http://wirtschaftslexikon.gabler.de/Archiv/14589/peer-group-v8.html

[68] Hildt, E., Franke, A.G. and Lieb, K. (2011) Pharmakologisches Neuroenhancement. Informationsquellen und Akzeptanzunter Studierenden. Nervenheilkunde, 30, 833 837.

[69] Kwan, P.P., Sussman, S. and Valente, T.W. (2015) Peer Leaders and Substance Use among High-Risk Adolescents. Substance Use \& Misuse, 50, 283-291. https://doi.org/10.3109/10826084.2014.977395

[70] World Anti-Doping Agency (WADA) (2015) The World Anti-Doping Code. The 2015 Prohibited List.

https://www.wada-ama.org/sites/default/files/resources/files/wada-2015-prohibitedlist-en.pdf

[71] Holland, A., Ed., Gedopte Profi-Gamer-Computerspieler müssen erstmals zum Drogentest. In: Augsburger Allgemeine, 8 August 2015.

[72] Brown, D. Performance Maintenance during Continuous Flight Operations, A Guide for Flight Surgeons (No. NAVMED P-6410). 1 January 2000.

http://www.med.navy.mil/directives/Pub/6410.pdf

[73] De Santis, A.D., Anthony, K.E. and Cohen, E.L. (2013) Illegal College ADHD Stimulant Distributors: Characteristics and Potential Areas of Intervention. Substance Use \& Misuse, 48, 446-56. https://doi.org/10.3109/10826084.2013.778281 


\section{Questionnaire on the prescription of methylphenidate (Ritalin ${ }^{\oplus}$ )}

\section{I am:}

a primary care physician

an internist

sth. different:

... and I have been working in the practice since:

1 to 5 years (since 2011)

5 to 10 years (before 2010)

... and I am practicing in:

10 to 15 years (before 2005)

Munich

Frankfurt

Augsburg

16 to 20 years (before 2000)

21 years and longer (before 1995)

Do you have patients in your care with prescribed methylphenidate for medical reasons?

No

Yes $\rightarrow \bigcirc$ Children

Adults

Have you ever had a case in which you were asked to prescribe methylphenidate without medical indication?

No, I never have

No, I never have, but I have colleagues who have

Yes, that has happened

$\rightarrow$ Only once

In total, three times at maximum

About once a month

First time years ago. 
If so: Who asked and who was it for?

An adult asked own use

$\rightarrow \bigcirc$ A known patient

An unknown patient

Age of 20 to 40 years

Age of 41 to 60 years

Over the age of 60 years

An adult asked in favor of a child

$\rightarrow \bigcirc$ A known patient

An unknown patient

What was the reason given - from your point of view - for requesting a prescription without indication?

ADHD in adults (but not proved by documents)

The primary care physician cannot be reached

The medicine is needed for learning and tests

The medicine is needed to improve the ability to work

No reason

What did you do when asked? (Multiple answers allowed)

A medical examination in order to verify whether the request is justified by medical indication

Called the alleged primary physician to ask whether the patient has a medical indication for methylpenidate

Referred the patient for clarification to a psychiatrist

Stated that I do not issue prescription without indication

Stated that I cannot write a prescription for narcotic drugs

I wrote a prescription for the patient

Other:

Do you want to add something related to the topic?

No

Yes:
Thank you for your cooperation.

You can return the questionnaire anonymous as well as personalized.

A stamped envelope is included.

stamp 
Fragebogen zur Verordnung von Methylphenidat (Ritalin ${ }^{\circledR}$ )

Ich bin:

Allgemeinarzt

Internist

Anderes:

... und praktiziere in:

München

Frankfurt

... in der Praxis tätig seit:

1 bis 5 Jahren (ab 2011)

5 bis 10 Jahren (vor 2010)

10 bis 15 Jahren (vor 2005)

16 bis 20 Jahren (vor 2000)

21 Jahre und länger (vor 1995)

Augsburg

Halle an der Saale

Haben Sie Patienten, die Methylphenidat aus medizinischen Gründen erhalten?

Nein

$\bigcirc \mathrm{Ja} \rightarrow \bigcirc$ Kinder

Erwachsene

Hatten Sie schon einmal den Fall, dass Sie ein Rezept für Methylphenidat ausstellen sollten, ohne dass hierfür eine medizinische Indikation bestand?

Nein, das hatte ich noch nie

Nein, das hatte ich noch nie, aber ich habe von Kollegen von solchen Fällen gehört

Ja, das kam schon einmal vor $\rightarrow$ ein einziges Mal

insgesamt nicht öfter als $3 \mathrm{Mal}$

etwa einmal pro Monat

das erste Mal vor Jahren 
Wenn ja: von wem und für wen wurde angefragt?
Ein Erwachsener wollte das
$\rightarrow \bigcirc$ Ein Patient von mir
Medikament für sich haben
Ein mir fremder neuer Patient
Alter 20 bis 40 Jahre
Alter 41 bis 60 Jahre
Alter über 60 Jahre
Ein Erwachsener wollte das
$\rightarrow \bigcirc$ Ein Patient von mir
Medikament für sein Kind haben
Ein mir fremder neuer Patient

Was war die Begründung für den aus Ihrer Sicht unbegründeten Rezeptwunsch?

ADHS des Erwachsenen (aber nicht durch Unterlagen belegt)

Der Hausarzt sei nicht erreichbar

Das Medikament werde zum Lernen oder für eine Prüfung benötigt

Das Medikament werde zum besseren Arbeiten benötigt

Keine Begründung

Was haben Sie gemacht, als die Anfrage kam? (Mehrfachantworten möglich)

Eine ärztliche Untersuchung, ob die Anfrage durch eine medizinische Indikation begründet ist

Beim vorgeblich behandelnden Arzt angerufen, ob es bei diesem Patienten eine Indikation für Methylphenidat gibt

Den Patienten zur Abklärung zu einem Psychiater überwiesen

Darauf verwiesen, dass ich ohne Indikation kein Rezept ausstelle

Darauf verwiesen, dass ich keine BtM-Rezepte habe

Dem Patienten ein Rezept ausgestellt

Anderes:

Haben Sie etwas, was Sie uns zu dem Thema mitteilen möchten?

Nein

Ja, und zwar:
Vielen Dank für Ihr Mitwirken.

Sie können diesen Fragebogen anonym oder namentlich zurückschicken.

Ein frankiertes Couvert liegt bei.

Stempel 
Submit or recommend next manuscript to SCIRP and we will provide best service for you:

Accepting pre-submission inquiries through Email, Facebook, LinkedIn, Twitter, etc. A wide selection of journals (inclusive of 9 subjects, more than 200 journals)

Providing 24-hour high-quality service

User-friendly online submission system

Fair and swift peer-review system

Efficient typesetting and proofreading procedure

Display of the result of downloads and visits, as well as the number of cited articles Maximum dissemination of your research work

Submit your manuscript at: http://papersubmission.scirp.org/

Or contact jbbs@scirp.org 